\title{
Parallel adaptations of Japanese whiting, Sillago japonica under temperature stress
}

\author{
Zhiqiang $\operatorname{Han}^{1}$, Xinyu Guo ${ }^{2}$, Qun $\mathrm{Liu}^{2}$, Shanshan $\mathrm{Liu}^{2}$, Zhixin Zhang ${ }^{3}$, shijun xiao ${ }^{4}$, and \\ Tianxiang $\mathrm{Gao}^{5}$ \\ ${ }^{1}$ Affiliation not available \\ ${ }^{2}$ BGI-Shenzhen \\ ${ }^{3}$ Tokyo University of Marine Science and Technology Graduate School of Marine Science \\ and Technology \\ ${ }^{4}$ Tibet Academy of Agricultural and Animal Husbandry Sciences \\ ${ }^{5}$ Zhejiang Ocean University
}

February 9, 2021

\begin{abstract}
Knowledge about the genetic adaptations of various organisms to heterogeneous environments in the Northwestern Pacific remains poorly understood. The mechanism by which organisms adapt to temperature in response to climate change must be determined. We sequenced the whole genomes of Sillago japonica individuals collected from different latitudinal locations along the coastal waters of China and Japan to detect the possible thermal adaptations. A total of 5.48 million single nucleotide polymorphisms (SNPs) from five populations revealed a complete genetic break between the China and Japan groups. This genetic structure was partly attributed to geographic distance and local adaptation. Although parallel evolution within species is comparatively rare at the DNA level, the shared natural selection genes between two isolated populations (Zhoushan and Ise Bay/Tokyo Bay) indicated possible parallel evolution at the genetic level induced by temperature. Our result proved that the process of temperature selection on isolated populations is repeatable. Additionally, the candidate genes were functionally related to membrane fluidity in cold environments and the cytoskeleton in high-temperature environments. These results advance our understanding of the genetic mechanisms underlying the rapid adaptations of fish species. Projections of species distribution models suggested that China and Japan groups may have different responses to future climate changes: the former could expand, whereas the latter may contract. The results of the present population genomic work expand our understanding of genetic differentiation and adaptation to changing environments.
\end{abstract}

Parallel adaptations of Japanese whiting, Sillago japonica under temperature stress

Zhiqiang Han ${ }^{1}$, Xinyu Guo ${ }^{2}$, Qun Liu ${ }^{2}$, Shanshan Liu², Zhixin Zhang ${ }^{3}$, Shijun Xiao ${ }^{4 *}$, Tianxiang $\mathrm{Gao}^{1^{*}}$

${ }^{1}$ Fishery College, Zhejiang Ocean University, Zhoushan, Zhejiang, 316002, China.

2 BGI-Qingdao, BGI-Shenzhen, Qingdao, Shandong, 266555, China

${ }^{3}$ Graduate School of Marine Science and Technology, Tokyo University of Marine Science and Technology, Konan, Minato, Tokyo, 108-8477, Japan

${ }^{4}$ Institute of Fisheries Science, Tibet Academy of Agricultural and Animal Husbandry Sciences, Lhasa, 850000, China

${ }^{*}$ Corresponding author. E-mail:shijun_xiao@163.com (Shijun Xiao), 
gaotianxiang0611@163.com (Tianxiang Gao)

\begin{abstract}
Knowledge about the genetic adaptations of various organisms to heterogeneous environments in the Northwestern Pacific remains poorly understood. The mechanism by which organisms adapt to temperature in response to climate change must be determined. We sequenced the whole genomes of Sillago japonica individuals collected from different latitudinal locations along the coastal waters of China and Japan to detect the possible thermal adaptations. A total of 5.48 million single nucleotide polymorphisms (SNPs) from five populations revealed a complete genetic break between the China and Japan groups. This genetic structure was partly attributed to geographic distance and local adaptation. Although parallel evolution within species is comparatively rare at the DNA level, the shared natural selection genes between two isolated populations (Zhoushan and Ise Bay/Tokyo Bay) indicated possible parallel evolution at the genetic level induced by temperature. Our result proved that the process of temperature selection on isolated populations is repeatable. Additionally, the candidate genes were functionally related to membrane fluidity in cold environments and the cytoskeleton in high-temperature environments. These results advance our understanding of the genetic mechanisms underlying the rapid adaptations of fish species. Projections of species distribution models suggested that China and Japan groups may have different responses to future climate changes: the former could expand, whereas the latter may contract. The results of the present population genomic work expand our understanding of genetic differentiation and adaptation to changing environments.
\end{abstract}

Keywords: Sillago japonica, local adaptation, climate change, temperature stress

\title{
Introduction
}

One of the fundamental questions in biology is how organisms adapt to diverse and changing environments (Schluter, 2000). Spatially varying selection usually triggers differential adaptations of local populations and ultimately initiates evolutionary diversification and speciation (Fustier et al., 2017; Ferchaud \& Hansen, 2016). Identification of the genetic basis for ecological adaptation is not only a primary goal in evolutionary biology, but it is also required to appropriately define conservation units (CUs) to help guide management and conservation efforts in changing environments (Chen et al., 2018; Skelly et al., 2007; Balanya, Huey, Gilchrist, \& Serra, 2009). Moreover, detecting candidate genes under natural selection can help identify the key gene pathways involved in adaptations to local environments. Marine environments are dramatically changing, and one of the most influential changes is rising temperature. Temperature represents a major environmental factor that influences spatial distribution and diversity of fish species (Chen, Farrell, Matala, Hoffman, \& Narum, 2018). Advancing our understanding of thermal adaptation is critical in predicting adaptive potential and ecological consequences of anthropogenic global warming.

Global climate change has considerable effects on organisms, especially for coastal species. In particular, the influence of climate change on marine organisms is reinforced in the East China Sea and the Yellow Sea, in which the water is warming at a higher rate than in other areas (Cai, Han, \& Yang, 2020). Simulation results showed that even under the low greenhouse emission scenario (i.e., RCP 4.5), the average annual sea surface temperature in the Yellow Sea would increase by at least $2{ }^{\circ} \mathrm{C}$ by the end of the 21 st century (http://www.bio-oracle.org/) (Assis et al., 2018). Therefore, climate-mediated selective signatures must be detected. Restriction site-associated DNA tags sequencing (RAD-seq) and genotyping-by-sequencing (GBS) methods have been applied in investigating the genetic adaptations of organisms in the Northwestern Pacific. However, these methods only cover a fraction of the total genome and may miss numerous loci under selection in local adaptations (Li, Xue, Zhang, \& Liu, 2018; Wang et al., 2016; Xu et al., 2017). Furthermore, the population genomics studies conducted in this region only revealed a possible signature of thermal adaptation, and no population-specific genome region or gene-related to warm or cold temperature has been analyzed. Adaptations to high or cold temperatures are expected to have a highly polygenic background, which is difficult to detect using reduced-representation sequencing genome scans. In addition to possible local adaptations, the similar distribution of ocean surface temperature between the coastal waters of China and Japan may lead to identical or similar adaptive changes in distantly independent populations, thereby 
causing parallel evolution.

Our recent population-scale genomic study on the Japanese whiting,Sillago japonica (Family Sillaginidae), demonstrated that this species is an ideal model for detecting signatures of parallel selection (Kashiwagi, Kondo, Yoshida, \& Yoshioka, 2000). This fish is a commercially important coastal species widely distributed throughout the Northwestern Pacific, especially in the East China Sea, the Yellow Sea, and the coastal waters of Japan (McKay, 1992; Oozeki, Hwang, \& Hirano, 1992). This species is euryhaline but is not observed to migrate over long distances (Yang, Gao, Meng, \& Jiang, 2020). GBS markers indicated substantial genetic differentiation between Chinese and Japanese populations, with Rushan (Weihai City, China) population as the transition population between China and Japan (Kashiwagi, Kondo, Yoshida, \& Yoshioka, 2000). These findings supported the supposition that the S. japonica populations in the East China Sea and the coastal waters of Japan are independent genetic populations. The Yellow Sea population that disperses from the East China Sea might be able to tolerate cold temperature stress during winter and induce adaptation to cold temperature on the genome. The west coast of East China Sea populations from China and some Japan populations encounter similar temperature stress that may cause parallel evolution and natural selection on the same genes. Therefore, S. japonica is an interesting species for studies of ecological adaptation because it inhabits diverse environments ranging from tropical to warm temperate climates, and it has low levels of genetic differentiation at the neutral loci (Gao, Yang, Yanagimoto, \& Xiao, 2019; Kashiwagi, Kondo, Yoshida, \& Yoshioka, 2000). The draft genome of S. japonica has been completed by the BGI-Shenzhen company (unpublished data). This draft genome provides a fundamental resource that enables the whole resequencing of genomes and the conduct of population genomic research.

In the present study, we sequenced the whole genome of 49 S. japonica individuals collected from five sites across the coastal waters of China and Japan that cover high-temperature (mean annual temperature $>$ 25 ), warm-temperature (mean annual temperature $>19$ ), and cold-temperature (mean annual temperature $>14$ ) areas. This study provides insights into the evolutionary history and genetic diversity of $S$. japonica , as well as an example of mechanisms by which a species can adapt to regions with different thermal environments. By comparing the genomes of S. japonica from cold- and high-temperature environments, we identified candidate genes with molecular functions that are potentially involved in local adaptations to temperature among $S$. japonica inhabiting different thermal environments. The comparison of $S$. japonica populations from the East China Sea and the coastal waters of Japan may provide possible evidence for parallel evolution within this species.

\section{Materials and Methods}

\section{Sampling}

We collected 49 individuals of $S$. japonica from five populations distributed throughout the coastal waters of China and Japan (Figure 1 and Table 1). All individuals were identified on the basis of morphological features. A piece of muscle tissue was obtained from each individual and preserved in $95 \%$ of ethanol or frozen for DNA extraction.

Sequencing, read mapping, and SNP calling

Genome sequencing was performed using the Illumina HiSeq 2500 platform by Novogene Bioinformatics Technology Co., Ltd. (Beijing, China). Quality control and removal of potential adaptor sequences from raw reads were performed to ensure the accuracy of bioinformatics analysis. Paired-end reads from each individual were aligned to the reference genome (unpublished data) using the Burrows-Wheeler Aligner (BWA) with the option "mem-t 4-k 32 -M" (Li \& Durbin, 2009). After mapping, the resulting bam files were sorted using SAMtools (Li, Handsaker, Wysoker, Fennell, \& Ruan, 2009), and duplicated reads were removed.

Raw SNPs were identified on the basis of mpileup files generated by SAMtools. The filtering threshold for raw SNPs was set to require a quality score of [?]20. Furthermore, an SNP was discarded if the total coverage of the SNP was less than one third or greater than five times the overall coverage. If two SNPs were $<5$ bp 
apart, both SNPs were removed.

\section{Genomic distribution of genetic variants}

ANNOVAR (Wang, Li, \& Hakonarson, 2010) was used to annotate the genomic distribution of variants and classify them into different categories (nonsynonymous, synonymous, UTR, $5 \mathrm{~kb}$ upstream, $5 \mathrm{~kb}$ downstream, intronic, and intergenic). The filter set for variants was a minor allele frequency of $>0.01$, depth of $>3$, and missing rate of $<0.2$.

\section{Population diversity and structure}

We used VCFtools (Danecek et al., 2011) to estimate nucleotide diversity (window size of $40 \mathrm{~kb}$ ) and the $F$ ST divergence statistic (window size of $40 \mathrm{~kb}$ ) for each pair of populations. We used Plink (Purcell et al., 2007) (http://pngu.mgh.harvard.edu/ purcell/plink/) to investigate the population structure, with ancestry clusters ranging from two to six. Principal component analysis (PCA) was performed using the GCTA software (Yang, Lee, Goddard, \& Visscher, 2011). The filtered SNP dataset was used to generate neighborjoining trees by using Treebest-1.9.2. Mantel tests with $F_{\mathrm{ST}} /\left(1-F_{\mathrm{ST}}\right)$ matrixes and distance matrixes were performed using the ade4 package (Dray \& Dufour, 2007) to test for isolation by distance. Geographical distances among samples were measured by following the coastline (coastal distances) and by the shortest distance across open waters (oceanic distances).

Demographic analysis

The demographic history of all five populations was analyzed using the PSMC model as implemented in the PSMC package (Li \& Durbin, 2011). In the absence of mutation rates from S. japonica or any closely related species, we used a mutation transition matrix based on medaka data (Spivakov et al., 2014). Point mutation rate and recombination rate per base were assumed to be $2.5 \times 10^{-8}$. Generation time was calculated as $g$ $=a+(s /[1-s])$, where $s$ is the expected adult survival rate, which is assumed as 80\%; and $a$ is sexual maturation age, which is one year for S. japonica. A generation time of 5 years was used. To determine the variance in the estimated effective population size, we performed 100 bootstraps for each population. Population-level admixture analysis was conducted using the TreeMix v.1.12 program (Pickrell \& Pritchard, 2012). The program inferred the ML tree for five populations. A window size of 1000 was used to account for linkage disequilibrium $(-\mathrm{k})$ and "-global" to generate the ML tree. Migration events $(-\mathrm{m})$ were sequentially added to the tree.

\section{Screening for signatures of selection}

To uncover the genetic variants involved in local adaptation of each population, we calculated the genomewide distribution of $F$ ST values and $\pi$ ratios for four control-thermal groups. These groups included the cold-temperature population RS versus the control groups (ZS and ST), the high-temperature population ST versus the control groups ( $\mathrm{RS}$ and $\mathrm{ZS}$ ), the China warm-temperature population ZS versus the control group (RS), and the Japan warm-temperature populations (IB and TB) versus the control group (RS).F ST and $\pi$ for sliding windows were calculated using VCFtools (Danecek et al., 2011), with a window size of $40 \mathrm{~kb}$ and a step size of $20 \mathrm{~kb}$. The windows with the top $5 \%$ values for the $F_{\mathrm{ST}}$ and $\pi$ ratios simultaneously served as the candidate outliers under strong selective sweeps. All outlier windows were assigned to their corresponding SNPs and genes. Overlapping genes within cold-, warm-, and high-temperature groups were selected for further analysis to avoid false positives. The selected genes in GO terms and KEGG pathways were enriched using OmicShare tools (http://www.omicshare.com/tools). Multiple comparisons were corrected using the FDR method (FDR-adjusted $P$ value $<0.05$ ).

Distribution prediction of China and Japan populations under climate change

We used maximum entropy (Phillips, Anderson, \& Schapire, 2006) (Maxent) model to predict potential distributions of two independent populations (China and Japan) under changing climates. We retrieved species presence records from Zhang et al. (2019) and divided them into China (22 recorders) and Japan (23 recorders) groups. On the basis of the results of Zhang et al. (2019) and the benthic life type of 
S. japonica, we considered five environment predictors from Bio-ORACLE (http://www.bio-oracle.org), including ocean depth, distance to shore, mean sea benthic temperature, salinity, and current velocity. The five predictors were not highly correlated (i.e., pairwise Pearson's correlation coefficient $|r|<0.70$ ) and thus all were used to develop Maxent models. Future (i.e., RCP 4.5 in 2100s [average for 2090 to 2100]) marine environmental predictors, including temperature, salinity, and current velocity, were also downloaded from Bio-ORALCE. We assumed that ocean depth and distance to shore would remain unchanged in the future. For each group, we developed Maxent model using all presence data of this group and present-day environmental predictors; this model was further used to predict potential distributions under present-day and future climatic conditions.

\section{Results}

We sampled 49 individuals from five populations covering the East China Sea, the Yellow Sea, and the Pacific coastal waters of Japan (Figure 1a and Table 1). Whole-genome resequencing yielded $680 \mathrm{~Gb}$ of sequencing data and generated $13.6 \mathrm{~Gb}$ for each individual. Genome alignment resulted in an average depth of 20.91-fold and an average of $99.40 \%$ genome coverage (at least one fold) (Supplementary Table S1). All individual data were aligned to the reference genome (mapping rate from $95.00 \%$ to $96.89 \%$ ) (Supplementary Table S2), and SNPs were called after rigorous quality filtering. We identified 5,483,086 SNPs after quality control for further analysis, with 406,925 in exonic regions, 2,025,230 in intronic regions, and 2,649,227 in intergenic regions. Of the exonic SNPs, we identified 312,482 synonymous and 94,443 nonsynonymous SNPs (Supplementary Table S3). The nucleotide diversity $\pi$ for each population was similar, ranging from $1.64 \times$ $10^{-3}$ to $1.69 \times 10^{-3}$ (Figure $1 \mathrm{~b}$ ).

Clustering analyses using PLINK was performed to examine the relationships among the populations (Figure 1c). The admixture analyses revealed clear evidence for clustering. With $K=2$, the admixture structure showed two clusters. Populations from China (RS, ZS, and ST) formed one cluster, whereas populations in Japan (IB and TB) formed another cluster. When $K=3$, the RS population formed a separate cluster. As the $K$ value increased, the ST population separated as a unique cluster. When $K$ increased from 3 to 6 , the ZS population was shared with clusters from RS and ST. TB and IB were highly admixed in all cases with $K$ from 2 to 6 . PCA results recovered the similar clusters achieved by the admixture analyses (Figure 1d). The first principal component of PCA (PC1) separated China and Japan clades, consistent with the admixture result at $K=2$. The second principal component of PCA (PC2) further separated RS, ZS, and ST populations but was not able to distinguish between TB and IB populations, consistent with the admixture results at $K=5$ and 6 (Figure 1c). All individuals from China groups clustered within the population defined by their sampling locations, revealing a signal of geographic structure from the East China Sea to the Yellow Sea.

The phylogenetic tree further supported the admixture analysis and PCA results. A neighbor-joining tree was constructed on the basis of whole-genome SNPs (Figure 1e). The tree formed two clades that represent the geographic divergence between the coastal waters of China and Japan. In the China clade, three distinct clusters generally defined by geographic localities were recovered. However, the phylogenetic topology of the Japan clade showed a shallow structure, and some of the individuals were grouped incorrectly into another geographic population.

We calculated pairwise $F$ ST between the populations to quantify their genetic differentiation (Table 2). Pairwise $F$ ST ranged from -0.0001 to 0.0377 , with an average of 0.0224 , consistent with the overall structured population. $F$ ST between China populations (RS, ZS, and ST) and Japan populations (IB and TB) ranged from 0.0237 to 0.0377 , with an average of 0.0316 . The level of genetic differentiation among the China populations was higher than that between the two Japan populations.

To explore the influence of geographic distance on genetic differentiation, we performed Mantel tests to test the association between the geographic distance matrix and the pairwise $F$ st matrix. Two patterns of geographic distance (i.e., coastal distance and oceanic distance) were used in the Mantel tests (Figure $2)$. We identified a closer relationship $(\mathrm{r}=0.90, P=0.0002)$ between $F_{S T} /\left(1-F_{S T}\right)$ and coastal distance 
than that between oceanic distance and $F_{S T} /\left(1-F_{S T}\right)(\mathrm{r}=0.80, P=0.0029)$, indicating isolation due to costal distance, with coastal distance explaining $91 \%$ of the variation in genetic differentiation for the species. These results of population structure analysis and Mantel tests collectively indicated that strong barriers might have had a greater influence on population differentiation than the other factors.

The demographic history of $S$. japonica was inferred to understand its evolutionary history. The historical effective population sizes of the populations were estimated via PSMC. Results showed that the demographic history of S. japonica could be traced back to approximately 100,000 years ago, and the China and Japan populations experienced evidently different demographic histories (Figure 3). Three China populations experienced similar demographic trajectories: one large population expansion and one population bottleneck. Three China populations suffered from an obvious population expansion approximately 30,000 years (Ka) ago. The effective population size of the China populations peaked $\sim 2-10 \mathrm{Ka}$, and then the effective population sizes, especially of the RS population, declined $\sim 2 \mathrm{Ka}$ and formed a bottleneck. Among the three China populations, the signal of population expansion increased from north to south, but the signal of bottleneck decreased from north to south. The IB and TB Japan populations underwent a different demographic history compared with the China populations: only one small population expansion. In contrast to the large and rapid population expansion of the China populations, the Japan populations started to increase slowly 10 Ka and reached the climax in the present.

We then reconstructed a maximum likelihood (ML) tree of the five populations by using TreeMix to address population history relationships and identify pairs of populations that are related to each other independent of that captured by this tree (Figure 4a). The ML without migration events inferred from the TreeMix analysis divided the 49 individuals into two clusters that were similar to the population structuring patterns identified from the phylogenetic tree analysis, PCA, and genetic structure analysis. When potential migration edges (i.e., migration events between the branches) were added to the ML tree, strong migration events were detected between the clusters (Figure $4 \mathrm{~b})$. We observed a statistically significant migration edge $(P<2.2 \mathrm{E}-$ 308 ) with the estimated weight of $28.8 \%$; this edge provided evidence for the gene flow from the Japan TB population into the RS population.

We compared the genomes of $S$. japonica individuals to identify signatures of positive selection under different environment selection pressures. Considering that genetic isolation occurred between the China and Japan populations, we chose the ST and ZS populations as the control groups to reveal candidate cold-temperature selection genes in the RS population. Using the top $5 \%$ maximum $F_{\mathrm{ST}}\left(F_{\mathrm{ST}}[?] \quad 0.1260\right)$ and $\pi$ ratio $\left(\Pi_{\mathrm{ST} / \mathrm{RS}}\right.$ [?] 1.2380) methods, a total of 132 candidate genes corresponding to $3.64 \mathrm{Mb}$ in size were identified in the RS population, with the ST population as the control group (Figure 5a). Using the ZS population as the control group, 509 candidate genes were selected corresponding to $18.96 \mathrm{Mb}$ in size $\left(F\right.$ ST [?] $0.0904, \Pi_{\mathrm{ZS} / \mathrm{RS}}$ [?] 1.2676). To validate further the candidate genes under strong selective sweeps in the RS population, we recognized a total of 81 genes in the overlap of RS/ZS and RS/ST pairs as potentially affected genes associated with cold-temperature adaptation for subsequent analyses (Figure 5b, Supplementary Table S4).

With the low-temperature populations RS and ZS as the control groups and using the top $5 \%$ maximum $F$ ST values $(F$ ST $[?] \quad 0.0485,0.0845)$ and $\pi$ ratio values $\left(\Pi_{\mathrm{RS} / \mathrm{ST}}[?] 1.2404, \Pi Z \mathrm{ZS}_{\text {ST }}[?] 1.1544\right), 1037$ and 534 candidate genes associated under selective sweeps in ST population were identified, respectively. A total of 365 genes shared by the ST/RS and ST/ZS pairs associated with high-temperature adaptation in the ST population were identified (Figure 5c, Supplementary Table S5). Only two cold-temperature adaptation genes overlapped with the high-temperature adaptation genes. Overall, 81 and 365 genes associated with cold- and high-temperature environments were positively selected, respectively.

To detect possible parallel adaptation between ZS and IB/TB populations, we identified 692 genes in ZS and 122 genes in Japan groups involved in warm-temperature adaptation, with the cold-temperature population RS as the control group. A total of 111 out of 122 (91.0\%) warm-temperature adaptation genes in the Japan populations overlapped with the warm-temperature adaptation genes of the ZS population (Supplementary Table S6), and no gene overlapped with the high-temperature adaptation genes of the ST population (Figure $5 \mathrm{~d})$. Considering the geographic isolation at the whole-genome SNPs and similar temperature environments, 
the highly shared selection genes between the Japan and ZS populations may be a possible evidence for parallel adaptive evolution.

The PCA results obtained on the basis of SNPs of the candidate genes related to cold-temperature adaptation showed that individuals from the RS population were correctly separated from the other populations (Figure 6a). PC1 tended to separate populations with different latitudes from south to north. PC2 separated the RS population from the other populations. Compared to high-temperature population, three warm-temperature populations (namely, ZS, IB, and TB) showed a closer relationship. Similarly, the PCA results that included the SNPs of the candidate genes for high-temperature adaptation separated the ST population (high temperature) from the other populations, with some individuals from the RS population at an intermediate position (Figure 6b). The allele frequency of one SNP within the cold-temperature adaptation gene Picalm was significantly associated with population temperature (Figure 5e). However, the allele frequencies of one SNP within the warm-temperature adaptation gene SORCS3 showed high values in three warm-temperature populations (Figure $5 \mathrm{f}$ ).

The PCA results that included the SNPs of the candidate genes from warm-temperature populations also divided the 49 individuals into two clades between the ST population and the other four populations, with the RS population at the intermediate position (Figure 6c). This topological pattern was different from that inferred from the genome-wide SNPs (Figure 1d), thus supporting the credibility of the candidate genes under selection. The substructure of the PCA based on the candidate parallel adaptation genes revealed a closer relationship between the ZS populations and IB/TB pair than with genome-wide SNPs, where the populations within the pair had large geographical and similar temperature environments (Figure 3, Table 1).

To obtain a broad overview of the molecular functions of these genes and detect the most differentiated regions of $S$. japonica genome, we performed GO enrichment and KEGG analyses on the candidate selection genes. These analyses offered a clear insight into the genetic evolution and adaptive mechanisms of $S$. japonica under different thermal environments.

The functional enrichment found that the candidate genes under cold selection were significantly enriched in only one KEGG pathway, that is, choline metabolism in cancer (ko05231, FDR adjusted $P=0.0421$ ) (Figure 7, Supplementary Table S7). The GO term enrichment analysis for the 81 candidate genes under cold temperature selection showed that the genes were classified into 47 categories, including metabolic processes, biological regulation, response to the stimulus, and signaling process in the biological process; membrane and membrane part in the cellular component; and binding and catalytic activity in the molecular function (Supplementary Figure S1). We found 50 significantly enriched GO terms after FDR correction (Supplementary Figure S2, Supplementary Table S8). The significantly enriched GO categories were primarily associated with cell part, cation transmembrane transporter activity, tissue homeostasis, transport, and lipid metabolism.

In the ST population, 365 genes were identified under selection, and these genes were significantly enriched in the cell adhesion molecule pathway (ko04514, FDR adjusted $P=0.0046$ ), tight junction pathway (ko04530, FDR adjusted $P=0.0189$ ), and leukocyte transendothelial migration (ko04670, FDR adjusted $P=0.0189$ ) (Figure 7, Supplementary Table S9). The selected genes were classified into 53 GO categories (Supplementary Figure S3), covering the major categories found in the RS population, including seven other categories (growth and behavior in the biological process; membrane-enclosed lumen in the cellular component; and transcription factor activity and protein binding in the molecular function). Four significantly enriched GO terms, namely, calcium-independent cell-cell adhesion via plasma membrane cell-adhesion molecules (GO: 0016338), establishment of the skin barrier (GO: 0061436), positive regulation of wound healing (GO: 0090303), and regulation of water loss via skin (GO: 0033561) were detected, all of which belong to the biological process (Supplementary Figure S2, Supplementary Table S10). Despite no significant GO terms in the cellular component and molecular function, the enriched categories in the cellular component and molecular function were mainly related to the cytoskeleton, the component of membrane, and transmembrane transporter activity. In this population, 13 genes in the cell-adhesion molecule pathway showed signals of 
natural selection. The 13 genes were nine members of Claudin family, JAM1, CDH4, and NLGN . Most members of the Claudin family were also located in the tight junction pathway and leukocyte transendothelial migration way. The enrichment of selected genes in the ST population suggested the importance of adaptation to high-temperature climate. The different patterns of enrichment for GO and KEGG pathways between the RS and ST populations may reflect the difference between cold- and warm-temperature stress.

A total of 111 genes were identified for parallel adaptations in the ZS, IB, and TB populations. These genes were classified into 48 categories (Supplementary Figure S4). Nineteen significantly overrepresented GO categories were detected for thermal adaptation (Supplementary Table S11). The GO clusters were primarily enriched in the categories of cell projection, structure of the cytoskeleton, binding, and maintenance of membrane (Supplementary Figure S2). Although no significantly enriched KEGG pathway was detected, the top 20 enriched pathways were related to metabolism (synthesis and degradation of ketone bodies, cyanoamino acid metabolism, linoleic acid metabolism, alpha-linolenic acid metabolism, and butanoate metabolism), circulatory system (vascular smooth muscle contraction), and endocrine system (salivary secretion and pancreatic secretion) (Figure 7, Supplementary Table S12). The enrichment of the selected genes in the warm-temperature population offered a parallel adaptive mechanism under thermal selection.

The receiver operating characteristic curve (AUC) results (0.983 in China group, 0.995 in Japan group) suggested that the Maxent model provides good predictive performance. The Maxent predictions for two groups of Japanese whiting suggested that the potential distribution of this species may greatly change (Figures 8 and 9). The predictions showed that a large part of coastal areas of China, central Japan, and Korea were suitable for the China group (Figure 8a). However, the more suitable area for the distribution of the Japan group was only in the southern and central Japan (Figure 9a). The northern boundary for the potential population distribution area of the Japan group in the East China Sea was Zhoushan. Climate change is predicted to influence the two groups differently. Future prediction for the China group under scenario RCP 4.5 showed that habitat suitability would clearly increase from south to north and the potential distribution may shift northward in the coastal waters of China and Japan by 2100 (Figure 8b,8c). However, the predicted result revealed a reduction in the potential area for the Japan group and no clear increase in the northern Japan areas by 2100. (Figure 9b,9c) This potential reduction in habitat suitability may indicate lower evolutionary potential to adapt to a changing environment. The response curves to temperature suggested different temperature adaptations between the two groups. The China group prefers high temperature, whereas the Japan group favors low temperature (Figure 8d,9d).

\section{Discussion}

\section{New insight on the genetic structure of $S$. japonica}

The historical and present variable environment has profound effects on the genetic variation of species. Analyses at the genomic level provide detailed information about the genetic structure, population history, and adaptation to various environments and facilitate species protection and fishery management (Li, Xue, Zhang, \& Liu, 2018). The present population genomic study delineated the fine population genetic characteristics of $S$. japonica populations via whole-genome sequencing. In the Northwestern Pacific, this study may be the first to adopt whole-genome sequencing in assessing population differentiation and signatures of selection in a marine fish species. Analyses of the population history suggested that the historical sea level has had a substantial influence on the effective population size of species, with a warmer climate facilitating population growth. Several genes related to adaptations to local environments were identified in the present study.

The present study provided a higher resolution of population structure compared with that identified in previous studies based on GBS, mtDNA control region, and morphological data (Gao, Yang, Yanagimoto, \& Xiao, 2019; Xue, Du, \& Gao, 2010). On the basis of the morphological analysis of S. japonica , Xue et al. found no significant population differentiation among populations from the Yellow Sea, East China Sea, and the South China Sea (Gao, Yang, Yanagimoto, \& Xiao, 2019). Researchers have attempted to use the mtDNA control region in finding genetic differentiation in this species. Gao et al. detected no genetic 
structure in S. japonica owing to the short fragment of this region (Gao, Yang, Yanagimoto, \& Xiao, 2019). However, using GBS technology, Yang et al. observed considerable genetic differentiation between the China and Japan populations ofS. japonica (Kashiwagi, Kondo, Yoshida, \& Yoshioka, 2000), but they failed to completely separate the individuals from these populations. In contrast to the mtDNA and GBS results of S. japonica, the present study revealed the complete genetic break between the China and Japan populations according to whole-genome sequencing data. PCA further distinguished the three China populations.

Numerous researchers have suggested that Pleistocene glaciations are the most important events that shaped the phylogeographic genetic structures of extant species (Han, Wang, Gao, Yanagimoto, \& Lida, 2018). The complete divergence between the China and Japan populations likely reflected historical isolation between the East China Sea and the Pacific Ocean during the Pleistocene low sea-level stands. In the present study, population demographic analysis suggested a divergence time of $30 \mathrm{Ka}$ between the two clades during the last glacial period. The dating of divergence was consistent with geological events that might have created a vicariant barrier between the S. japonicapopulations of the Pacific Ocean and the East China Sea.

Mantel tests identified a strong relationship between coastline distance and genetic differentiation. The ocean distance that separates the western and eastern East China Sea is identified as a physical barrier that restricts gene flow between samples. Considering the life history of this species and the physical environment in the East China Sea, long ocean distance is a reasonable physical barrier for a demersal species. Ocean depth $(<30 \mathrm{~m})$ limits the distribution of $S$. japonica in marine waters (FishBase,https://www.fishbase.in/summary/Sillago-japonica.html). The average depth of the East China Sea is about, with a maximum of at the continental slope (Guan, \& Mao, 1982). Therefore, the depth of water in the direct dispersal route between the coastal waters of Japan might have formed an unsuitable habitat for this species and prevented its offshore dispersal. The historical migration event from the TB to RS populations and no migration from the Japan populations to the ZS population supported the coastal dispersal pattern. The coastal dispersal pattern was also supported by the potential distribution areas of two groups as predicted by Maxent model. The coastal dispersal pattern was also observed in Japanese grenadier anchovy, Coilia nasus (Gao, Wan, Song, Zhang, \& Han, 2014; Han, Han, Wang, \& Gao, 2015). C. nasusshares similar biological characteristics and geographic distribution in the East China Sea with $S$. japonica. AFLP and mtDNA results of C. nasus confirmed that direct ocean distance with deepwater at the continental slope between the western and eastern coastal waters of the East China Sea served as a major physical barrier to this species.

The different demographic trajectories of the S. japonicapopulations might have resulted from geographic and climatic differences. Moreover, the TB and IB populations showed a slower increase compared with the China populations during the same time. The Japan populations (TB and IB) were mainly distributed in the coastal waters of Japan and thus might have suffered less impact during the Pleistocene glaciations. The expansion of population size in the China and Japan populations was consistent with the historical sea-level rise during the interglacial periods. The population started to increase dramatically about $30 \mathrm{Ka}$ before the present when the Wurm glacial stage began to end in the Northwestern Pacific (Kawahata \& Ohshima, 2004).

Previous studies revealed that marine pelagic and demersal fishes in the coastal waters of China are expected to exhibit little intraspecific genetic structuring derived from the ocean currents and the apparent lack of physical barriers (Han, Gao, Yanagimoto, \& Sakurai, 2008). Given the wide distribution and ecological characters of $S$. japonica, this species might be susceptible to a heterogeneous local environment. Likewise, genome-wide SNPs revealed remarkable regional differentiation between northern and southern populations in recent genomic studies of the roughskin sculpin (Trachidermus fasciatus), the marbled rockfish (Sebastiscus marmoratus ), the spotted seabass (Lateolabrax maculatus), and the small yellow croaker (Larimichthys polyactis ) (Li, Xue, Zhang, \& Liu, 2018; Xu et al., 2017; Liu, Zhang, Xue, Gao, \& Liu, 2016). In this sense, the high degree of regional differentiation between the northern and southern Chinese populations might be common in marine fish species owing to local adaptations to a heterogeneous environment. For instance, the average annual sea surface temperature ranges from $15.9{ }^{\circ} \mathrm{C}$ in the RS population to $25.2{ }^{\circ} \mathrm{C}$ in the $\mathrm{ST}$ 
population (data provided by the Bio-ORACLE). This obvious difference in the thermal environment might have resulted in divergent selection on specific genes. The genetic diversity in certain genome regions would be substantially decreased as a result of natural selection.

\section{Adaptive mechanisms for cold temperature}

A good understanding of how ecological variants of fishes can affect their population structure will provide more comprehensive implications for conservation management and decision-making (Li, Xue, Zhang, \& Liu, 2018). Water temperature is one of the most important abiotic factors that influence the phenotypes and habitats of aquatic organisms (Chen, Farrell, Matala, Hoffman, \& Narum, 2018). As an important environmental stressor, low and high temperatures have broad biological effects on marine organisms. These thermal effects generate intense selective pressures on several genes and genome regions.

In this study, selective sweep was conducted to identify the thermally affected genes between the northern and southern populations of S. japonica. The strategy of combining alternative statistical approaches in detecting selective signatures can provide sound results by decreasing false-positive rates. We evaluated the genetic attributes of the candidate genes relative to the genomic background. We scanned the genome-wide variations using $F$ ST and $\pi$ ratio values. The cold- and high-temperature adaptation conditions shared only two candidate genes.

A total of 81 candidate genes shared by both the $\pi$ ratio and $F$ ST analysis in the RS population were recognized as potentially affected genes related to cold adaptation. Cold adaptation and acclimatization studies suggested that more than one mechanism is involved in the biological response to cold stress (White, Alton, \& Frappell, 2012; Liu et al., 2018). Consistent with our expectations from the biological complexity of cold adaptation, several different processes, rather than one particular term or pathway, were highlighted by our selection tests. Low temperatures may influence energy metabolism. Nine selection genes (low-density lipoprotein receptor $[L D L R], Z B T B 20, P I C A L M$, and nup35) were related to lipid metabolic process. Lipids are the main components of cytomembrane (Meer, Voelker, \& Feigenson, 2008). A common cold adaptation mechanism for the cell is to manipulate the membrane lipid composition to maintain membrane fluidity and, correspondingly, proper membrane permeability and function of membrane protein complexes (e.g., transporters) (Russell \&Nichols, 1999).

Previous studies showed that the permeability of cell membranes considerably changes under long-term low temperature conditions and damages the integrity and stability of cell membranes. The LDLRgene is one important candidate gene for cold adaptation. $L D L R$, a glycoprotein located on the surface of cells, mediates the endocytic uptake of LDL cholesterol in the liver, which is a key regulator of the metabolism of plasma low-density lipoprotein cholesterol. $L D L R$ is the most powerful determinant of variation in total cholesterol and LDL cholesterol levels (Hansen et al., 1997). This gene plays an essential role in protecting cell membrane integrity under cold stress. The strong selection pressure on this gene is useful in adapting to low temperatures. A similar result was also observed in the low-density lipoprotein receptor-related protein 5 (LRP5 ) gene, another gene member of low-density lipoprotein receptor of humans (Cardona et al., 2014). LRP5 shows strong signals of selection in indigenous Siberian human populations. LRP5 has a high expression in the liver of humans and plays a role in cholesterol metabolism. Natural selection of the LRP5 gene helps Siberian persons to cope with the cold climate. The TPO gene is involved in thyroid metabolism (Leonard, Snodgrass, \& Sorensen, 2005). The thyroid determines the basal metabolic rate of the body. Natural selection of the TPO gene in the RS population may maintain the basal metabolic rate and stabilize lipid levels in the serum.

We also detected substantial adaptive evidence concerning ion exchange and transportation, which are processes that affect the fluidity and permeability of the cell membrane and directly and indirectly linked to thermal regulation. We identified a considerable number of genes that encode transporters (the MFS transportersSLC22A5 , SLC7A2 , and SLC25A5 ) and ion channels (e.g., the voltage-gated sodium channel $S C N 4 B$ ) in the genome regions of the RS population under selective sweeps. For example,SLC22A5 is a specific transporter that exists in the membranes of cells and mitochondria involved in the uptake or release 
of carnitine. Four copies of SLC22A5 in the genome of S. japonicaindicated gene expansion. Carnitine is a carrier for long-fatty acids and facilitates their transport into the mitochondria for lipid oxidation. Defective SLC22A5 causes systemic carnitine deficiency, resulting in metabolic decompensation. SLC7A2functions as a permease involved in transporting cationic amino acids across the plasma membrane. These transporter and ion channel genes are crucial for transmembrane transport to maintain a stable balance between the internal and external environments of cells under cold temperature. Therefore, the genes that encode transcellular ion transporters and channel proteins are reshaped by natural selection under cold stress environment.

Smooth muscle contraction, which includes vasoconstriction and vasodilation, is another process implicated in cold adaptation. Two genes involved in this process that showed evidence of strong signals of selection are CPI17 (protein phosphatase 1 regulatory subunit 14A) and CACNB4 . CPI17 is the inhibitor of PPP1CA. It has more than 1000-fold inhibitory activity when phosphorylated, creating a molecular switch for regulating the phosphorylation status of PPP1CA substrates and smooth muscle contraction in the absence of increased intracellular $\mathrm{Ca}^{2+}$ concentration ( $\mathrm{Li}$ et al., 2001). The CACNB4 gene encodes a calcium channel subunit expressed in the heart and increases peak calcium current that is important for cardiac muscle contraction under cold stress (Rouhiainen et al., 2016). Although no data on the heart rate of the populations are available, cold exposure is known to increase cardiac pressure. Thus, this efficient cardiovascular regulation might be a possible cold adaptive mechanism in the RS population. These genetic changes might facilitate the adaptation and survival of $S$. japonica in low-temperature areas.

Cell repair and clear necrotic organelles are also important processes of cold adaptation in the RS population. Interferon regulatory factor 1 (IRF1) is a transcriptional regulator that displays a remarkable functional diversity in regulating cellular responses (Oshima et al., 2004). Under cold stress, IRF1 causes cells to suspend proliferation to survive in adverse environmental conditions and also decisively triggers apoptosis when cell damage becomes irreparable, thereby preventing harmful cells from harming other normal cells. DnaJ homolog subfamily C member 10 is involved in the correct folding of proteins and the degradation of misfolded proteins (Oka, Pringle, Schopp, Braakman, \& Bulleid, 2013). It promotes the apoptotic signaling pathway in response to endoplasmic reticulum stress.

The enrichment tests provided 50 significant GO terms and one KEGG pathway after FDR adjustment. The enrichment GO categories and KEGG pathways were primarily associated with cell apart, transmembrane transporter activity, tissue homeostasis, lipid metabolism, apoptosis, and vascular smooth muscle contraction. These functional clusters are biologically relevant to cold adaptations and essential in regulating mechanisms for fish to respond to a cold environment.

\section{Adaptive mechanisms for high temperature}

In the ST population, different mechanisms were involved in the biological response to high temperature. Cells increase the baseline of some proteins to adapt to high water temperature. RAB6A and DDX52 were found under high selection pressures in the ST population. These genes are responsible for intracellular protein transport, retrograde vesicle-mediated transport of Golgi apparatus to endoplasmic reticulum, and ATP-dependent RNA helicase activity, a response that reflects the protein turnover stimulated by heat shock (Saadeldin et al., 2020).

Chronic thermal stress causes damaging effects on the cytoskeleton, catalytic activities of enzymes, singleand double-stranded DNA breaks and mutations, and inhibition of the DNA repair pathway, leading to apoptosis and necrosis (Saadeldin et al., 2020). Genome-wide selective sweep analysis of the ST population revealed that numerous genes embedded in selected genome regions belonged predominantly to functional categories related to paracellular permeability, including cell junction (GO: 0030054), bicellular tight junction (GO: 0005923), apical junction complex (GO: 0043296), cell-cell junction (GO: 0005911), and occluding junction (GO: 0070160) (Supplementary Table S8), as well as tight junction pathway (KEGG: 04512), cell adhesion molecule pathway (KEGG:04514), and its downstream pathway leukocyte transendothelial migration (KEGG:04670). We identified several essential genes, includingLRAD4, PAK3 , BCAS3 , MINK1, PDLI4 ,RTN4RL1, CLAUDIN (CLDN3 , CLDN4, and CLDN6 ),CDH4, NLGN3 , and JAM1, within 
the genome regions under selective sweeps. When exposed to heat stress, cells respond by dramatically reorganizing various cytoskeletal networks, such as microtubules, intermediate filaments, and actin microfilaments. TheLRDA4 gene serves as a negative regulator of TGF-beta signaling. The TGF-beta signaling pathway is related to cytoskeleton organization and involved in morphological changes associated with cellular responses to chronic heat (Saadeldin et al., 2020). Therefore, LRAD4prohibits the expression of the TGF-beta signaling pathway to maintain cell morphology during heat stress. The PAK3 , BCAS3 ,MINK1 , PDLI4, and RTN4RL1 genes play an important role in cytoskeleton regulation to ensure cytoskeletal integrity under chronic heat shock. $P A K 3$ plays a role in various signaling pathways, including cytoskeleton regulation, cell migration, and cell cycle regulation (Boda et al., 2004).

Cadherin, Claudin, and Jam1 are the key components of the tight junctions of KEGG pathways. The tight junction maintains the integrity of the epithelial cell layer that protects multicellular organisms under external stress and stimuli (Anderson \& Itallie, 2009). The tight junction also serves as a physical barrier to prevent solutes and water from passing freely through the paracellular space between epithelial and endothelial cell sheets. Cell dehydration is an important cause of high-temperature injury. Claudin 4 , a member of the Claudin family, was located in two significant GO terms (establishment of the skin barrier, GO: 0061436; regulation of water loss via skin, GO: 0033561) that are related to water homeostasis.

Moreover, aside from negatively affecting the cytoskeleton and cytomembrane integrity, high-temperature stress usually causes DNA damage and apoptosis. We found that ATRX, RFC3 ,Npas2, and RFA1 were under higher selection pressures in the ST population than in the ZS and RS populations. These genes are involved in DNA repair or cellular response to DNA damage stimulus (Juhász, Elbakry, Mathes, \& Löbrich, 2018; Xia, Xiao, Gannon, \& Li, 2010; Lisby, Barlow, Burgess, \& Rothstein, 2004). Four genes, namely, BNIP1 ,AIFM1 , AIFM3 , and Taok1, were in the GO term execution phase of apoptosis (GO: 0097194). Cells die as the execution phase is completed. AIFM1 and AIFM3, which are apoptosis-inducing factors, trigger chromatin condensation and DNA fragmentation in cells to induce programmed cell death.

Heat stress usually changes membrane permeability, thereby increasing intracellular $\mathrm{Na}^{+}$and $\mathrm{Ca}^{2+}$ (Park, Han, Oh, \& Kang, 2005). The TRPC4 channel is involved in various physiological and pathophysiological processes, such as vascular smooth muscles, endothelial functions, adiponectin regulation, and oxidative stress. TRPC4 has an important role in regulating cytosolic calcium ion concentration in mice and interferes with actin expression (Yang et al., 2005). High temperature causes endoplasmic reticulum physiological dysfunction and calcium homeostasis imbalance. High temperature also induces unfolded and misfolded proteins to accumulate in the endoplasmic reticulum in excess, thereby triggering the endoplasmic reticulum stress response. Four genes, namely,DNAJB14, AIFM1 , CREBRF , and Stc2 , are part of the endoplasmic reticulum stress response. ER stress responses include unfolded protein response, which is initially aimed at compensating for damage but can eventually trigger cell death if endoplasmic reticulum dysfunction is severe or prolonged. DNAJB14acts as a co-chaperone of HSPA8/Hsc70 in promoting protein folding and trafficking, as well as in promoting unfolded proteins to the endoplasmic reticulum-associated degradation pathway. AIFM1 functions as an NADH oxidoreductase and a regulator of apoptosis. It interacts with EIF3G , thereby inhibiting the EIF3 machinery and protein synthesis. Moreover, it activates caspase-7 to amplify apoptosis. Furthermore, DNAJB14 , CCT6 , CCT8 , andDNAJB13 are important molecular chaperones that assist in the folding of proteins upon ATP hydrolysis, thereby preventing or correcting misfolding caused by high temperature.

Thus, natural selection has substantially changed genetic variations in a vast number of genes in the ST population related to the regulation of the cytoskeleton, cell morphogenesis, ion transmembrane transport, DNA repair, protein folding, and apoptosis. These processes may maintain the integrity of cells and homeostasis, allowing the fitness of $S$. japonica to increase under high-temperature stress.

\section{Evidence of parallel evolution in several warm-temperature adaptation genes}

In general, geographically distinct populations that are exposed to similar environmental conditions evolve similar genotypic and phenotypic traits. The replicated nature of $S$. japonica provides clear molecular si- 
gnatures that can be used to recover many alleles consistently associated with parallel warm-temperature adaptations between the ZS and IB/TB populations. In the present study, most genes (91.0\%) associated with local warm-temperature adaptation in the Japan populations were shared with selection genes in the ZS population, in which the populations spanned long geographic distances and showed a similar annual temperature (19.1 -21.3). However, the selection genes in the Japan populations did not overlap with the high-temperature adaptation genes in the ST population. The integration of the shared selection genes and similar temperature environments could be considered as a possible evidence for parallel evolution in $S$. japonica. The PCA results based on the SNPs of the shared selection genes revealed close but distinguishable patterns between the ZS and two Japan populations, indicating independent selection events on the same genes between the ZS and IB/TB populations. A similar conclusion was reported for the three spine stickleback. Fixation of different loci at the same gene was observed in marine-freshwater divergence of threespine stickleback (Barrett, Rogers, \& Schluter, 2008). Evidence of parallel evolution at the TSHR gene was observed between the Atlantic herring populations from both sides of the Atlantic Ocean. TSHRis a major gene associated with the timing of reproduction (Lamichhaney et al., 2017). Parallel genetic evolution was also reported in the Atlantic cod and Sebastiscus marmoratus (Xu et al., 2017; Bradbury et al., 2010).

In the present study, S. japonica provided an opportunity to study the genetic basis of repeated parallel evolution in geographically distant populations. The results offered a chance to identify the warm-temperature adaptation genes in different independent populations. Candidate genes were identified within peaks of parallel divergence between the ZS and IB/TB populations. These candidate genes may be important for adaptation to warm temperature. The GO clusters were primarily enriched in the categories of cell projection, structure of the cytoskeleton, protein binding, maintenance of membrane, and cell differentiation. However, no significantly enriched KEGG pathways were detected. The top 20 enriched pathways were related to metabolism (synthesis and degradation of ketone bodies, cyanoamino acid metabolism, linoleic acid metabolism, alpha-linolenic acid metabolism, and butanoate metabolism), circulatory system (vascular smooth muscle contraction), and endocrine system (salivary secretion and pancreatic secretion).

Given that $S$. japonica is a species adapted to warm temperatures, the warm temperature had a relaxed selective pressure on the genes involved in response to heat stress. Moreover, the warm temperature promoted the growth of $S$. japonica by increasing the fluidity of the cell membrane and requiring rapid cell division and frequent extracellular and intracellular exchange of substances. Among the candidate genes for warmtemperature adaptation, four genes were located in the GO term cell division (GO: 0051301), 17 genes were involved in the cytoskeleton (GO: 0016328), 10 genes were located in GO term transmembrane signaling receptor activity (GO: 0004888), and 8 genes were located in GO term ion transmembrane transport (GO: 0034220). Various receptors in the membrane and ion transmembrane transport genes have important roles in the material exchange between the inside and outside environments of cells to satisfy the requirement for rapid cell division. Furthermore, three genes (FMN2 , MGMT, and POLH ) were located in the GO term DNA repair (GO: 0006281), thereby avoiding DNA replication errors. FMN2 plays a role in responding to DNA damage, cellular stress, and hypoxia by protecting CDKN1A against degradation. FMN2 also promotes the assembly of nuclear actin filaments in response to DNA damage to facilitate the movement of chromatin and repair factors after DNA damage (Yamada, Ono, Perkins, Rocha, \& Lamond, 2013). MGMT is crucial for genome stability. It repairs the naturally occurring mutagenic DNA lesion O6-methylguanine back to guanine and prevents mismatch and errors during DNA replication and transcription (Kawate et al., 2000). POLHserves as a DNA polymerase specifically involved in DNA repair by translesion synthesis (Masutani et al., 1999). Warm temperature promotes material exchange, DNA replication, and cell division. Suitable temperature ensures that enough food resources are available in the environment. Hence, these terms are functionally necessary for the adaptations of $S$. japonica to warm temperatures.

\section{Different climate resilience between China and Japan groups}

Natural selection analysis demonstrated that different populations have differential temperature adaptive capacity at the genetic level. We also obtained evidence of differences in temperature adaptation between the China and Japan populations at the macro level through species distribution model. The different change 
in the potential distributions for two groups under climate change may reflect the different thermal ability between the China and Japan groups. The China group contains different thermal adaptation populations and may have strong adaptive capacity adaptation to future climate change. However, the Japan group with stenothermal character shows a weak adaptive capacity to climate change. Our finding is different from the predicted distribution of this species previously reported by Zhang et al. (2019), who did not consider genetic differences within this species. Cryptic diversity clearly affects global climate change projections. In a previous study, Balint et al. (2011) showed that without discerning intraspecific genetic variation and cryptic diversity, the effects of global climate change were likely to be drastically underestimated. From our findings, the combination of phylogeographic data and species distribution model provide new understanding of the evolutionary trajectories of species under changing environmental conditions.

\section{Conclusion}

This study confirmed the existence of two genetically differentiated populations that showed strong parallel evolution. Such genetic parallelism was likely due to similar temperature environments. We identified population-specific genes that may be related to adaptations to high and low temperatures. These genes can be potential candidates for further exploration of the underlying mechanisms involved in confronting various challenges. In conclusion, by comparing the whole genomes of $S$. japonica populations from environments with different temperatures, we revealed various genes associated with local adaptations of this species to cold- and high-temperature environments. Specifically, the candidate genes were functionally related to membrane fluidity in cold environments and the cytoskeleton in high-temperature environments. These results advance our understanding of the underlying genetic mechanisms that allow rapid adaptations of fish species, particularly small ruminants, to survive in extreme environments.

\section{Data availability statement}

The whole-genome sequence raw reads for S. japonica have been deposited in the NCBI Sequence Read Archive under the accession number *.

\section{Ethics statement}

All animal work was conducted according to a permit (no. ZJOUFS-20181106) approved by the Committee for Animal Experiments of Fishery College, Zhejiang Ocean University.

\section{Acknowledgments}

We thank Shengyou Xu (Zhejiang Ocean University) and Fangrui Lou (Ocean University of China) for valuable suggestions and comments. The research was funded by the National Natural Science Foundation of China (41976083 and 41776171).

\section{Author Contributions}

X.S.J. and T.X.G. conceived and supervised the study. T.X.G. performed sample collection. X.Y.G. conducted DNA extraction, genome sequencing. Q.L. conducted SNP calling and genetic diversity analysis. S.S.L. performed PCA analysis. Z.Q.H. performed analyses of population genetic structure, demographic history, signatures of selection, and wrote the draft manuscript. Z.X.Z performed species distribution model analysis.

\section{SUPPLEMENTARY MATERIALS}

Figure S1 GO secondary node annotation classification of cold-temperature adaptation genes

Figure S2 Top 20 results of the enrichment analysis of GO terms for candidate genes. (a-c, cold-temperature adaptation genes; $\mathrm{d}-\mathrm{f}$, high-temperature adaptation genes; $\mathrm{g}-\mathrm{i}$, warm-temperature adaptation genes).

Figure S3 GO secondary node annotation classification of high-temperature adaptation genes

Figure S4 GO secondary node annotation classification of warm-temperature adaptation genes 
Table S1 Statistics of whole-genome sequencing data output

Table S2 Sequencing depth and coverage statistics

Table S3 SNP summary statistics in Sillago japonica

Table S4 List of cold-temperature adaptation genes and related annotation information

Table S5 List of high-temperature adaptation genes and related annotation information

Table S6 List of warm-temperature adaptation genes and related annotation information

Table S7 KEGG enrichment analysis of cold-temperature adaptation genes

Table S8 GO enrichment analysis of cold-temperature adaptation genes

Table S9 KEGG enrichment analysis of high-temperature adaptation genes

Table S10 GO enrichment analysis of high-temperature adaptation genes

Table S11 GO enrichment analysis of warm-temperature adaptation genes

Table S12 KEGG enrichment analysis of warm-temperature adaptation genes

\section{Reference}

Anderson, J. M., \& Itallie, C. M. V. (2009). Physiology and function of the tight junction. Cold Spring Harbor Perspectives in Biology ,1 (2), a002584.https://doi.org/10.1101/cshperspect.a002584

Assis, J., Tyberghein, L., Bosch, S., Verbruggen, H., Serrao, E. A., \& Clerck, O. D. (2018). Bio-ORACLE v2. 0: Extending marine data layers for bioclimatic modelling. Global Ecology and Biogeography ,27 (3), 277-284.https://doi.org/10.1111/geb.12693

Balanya, J., Huey, R. B., Gilchrist, G. W., \& Serra, L. (2009). The chromosomal polymorphism of Drosophila subobscura : a microevolutionary weapon to monitor global change. Heredity ,103 (5), 364367.https://doi.org/10.1038/hdy.2009.86

Balint, M., Domisch, S., Engelhardt, C. H. M., Haase, P., Lehrian, S., Sauer, J., ... Nowak, C. (2011). Cryptic biodiversity loss linked to global climate change. Nature Climate Change , 1 (6), 313318.https://doi.org/10.1038/nclimate1191

Barrett, R. D. H., Rogers, S. M., \& Schluter, D. (2008). Natural selection on a major armor gene in threespine stickleback.Science , 322 (5899), 255-257.https://doi.org/10.1126/science.1159978

Boda, B., Alberi, S., Nikonenko, I., Node-Langlois, R., Jourdain, P., Moosmayer, M., ... Muller, D. (2004). The mental retardation protein PAK3 contributes to synapse formation and plasticity in hippocampus. Journal of Neuroscience, 24 (48), 10816-10825.https://doi.org/10.1523/JNEUROSCI.2931-04.2004

Bradbury, I. R., Hubert, S., Higgins, B., Borza, T., Bowman, S., \& Paterson, I. G. (2010). Parallel adaptive evolution of Atlantic cod on both sides of the Atlantic Ocean in response to temperature.Proceedings of the Royal Society B-Biological Sciences, 2777 (1701), 3725-3734.https://doi.org/10.1098/rspb.2010.0985

Cai, R. S., Han, Z. Q., \& Yang, Z. X. (2020). Impacts and risks of changing ocean on marine ecosystems and dependent communities and related responses. Advances in Climate Change Research ,16 (2), 182-193.

Cardona, A., Pagani, L., Antao, T., Lawson, D. J., Eichstaedt, C. A., Yngvadottir, B., ... Kivisild, T. (2014). Genome-Wide Analysis of Cold Adaptation in Indigenous Siberian Populations. PLoS One ,9 (5), e98076.https://doi.org/10.1371/journal.pone.0098076

Chen, C., Wang, H. H., Liu, Z. G., Chen, X., Tang, J., .. Shi, W. (2018). Population genomics provide insights into the evolution and adaptation of the eastern honey bee (Apis cerana). Molecular Biology and Evolution , 35 (9), 2260-2271.https://doi.org/10.1093/molbev/msy130 
Chen, Z. Q., Farrell, A. P., Matala, A., Hoffman, N., \& Narum, S. R. (2018). Physiological and genomic signatures of evolutionary thermal adaptation in redband trout from extreme climates. Evolutionary Applications , 11 (9), 1686-1699.https://doi.org/10.1111/eva.12672

Danecek, P., Auton, A., Abecasis, G., Albers, C. A., Banks, E., DePristo, M. A., ... Durbin, R. (2011). The variant call format and VCFtools. Bioinformatics , 27 (15), 21562158.https://doi.org/10.1093/bioinformatics/btr330

Dray, S., Dufour, A. B. (2007). The ade4 package: Implementing the duality diagram for ecologists. Journal of Statistical Software ,22 (4), 1-20.http://dx.doi.org/10.18637/jss.v022.i04

Ferchaud, A. L., \& Hansen, M. M. (2016). The impact of selection, gene flow and demographic history on heterogeneous genomic divergence: three-spine sticklebacks in divergent environments. Molecular Ecology , 25 (1), 238-259.https://doi.org/10.1111/mec.13399

Fustier, M. A., Brandenburg, J. T., Boitard, S., Lapeyronnie, J., Eguiarte, L., Vigouroux, Y., ... Tenaillon, M. I. (2017). Signatures of local adaptation in lowland and highland teosintes from whole-genome sequencing of pooled samples. Molecular Ecology ,26 (10), 2738-2756.https://doi.org/10.1111/mec.14082

Gao, T. X., Wan, Z. Z., Song, N., Zhang, X. M., \& Han, Z. Q. (2014). Evolutionary mechanisms shaping the genetic population structure of coastal fish: insight from populations of Coilia nasus in Northwestern Pacific. Mitochondrial DNA , 25 (6), 464-472.https://doi.org/10.3109/19401736.2013.814109

Gao, T. X., Yang, T. Y., Yanagimoto, T., \& Xiao, Y. S. (2019). Levels and patterns of genetic variation in Japanese whiting (Sillago japonica) based on mitochondrial DNA control region. Mitochondrial DNA A, 30 (1), 172-183.https://doi.org/10.1080/24701394.2018.1467411

Guan, B. X., \& Mao, H. L. (1982). A note on circulation of the East China Sea. Chinese Journal of Oceanology and Limnology ,1 (1), 5-16.https://doi.org/10.1007/BF02852887

Han, Z. Q., Gao, T. X., Yanagimoto, T., \& Sakurai, Y. (2008). Deep phylogeographic break among white croaker Pennahia argentata(Sciaenidae, Perciformes) populations in North-western Pacific.Fisheries Science , 74 (4), 770-780.https://doi.org/10.1111/j.1444-2906.2008.01588.x

Han, Z. Q., Han, G., Wang, Z. Y., \& Gao, T. X. (2015). The possible physical barrier and coastal dispersal strategy for Japanese grenadier anchovy, Coilia nasus in the East China Sea and Yellow Sea: Evidence from AFLP Markers. International Journal of Molecular Sciences ,16 (2), 32833297.https://doi.org/10.3390/ijms16023283

Han, Z. Q., Wang, Z. Y., Gao, T. X., Yanagimoto, T., \& Lida, K. (2018). Assessing the speciation of a cold water species, Japanese sand lanceAmmodytes personatus, in the Northwestern Pacific by AFLP Markers. Animals , 8 (12), 224.https://doi.org/10.3390/ani8120224

Hansen, P. S., Defesche, J. C., Kastelein, J. J., Gerdes, L. U., Fraza, L., Gerdes, C., ... Schuster, H. (1997). Phenotypic Variation in Patients Heterozygous for Familial Defective Apolipoprotein B (FDB) in Three European Countries. Arteriosclerosis Thrombosis and Vascular Biology , 17 (4), 741747.https://doi.org/10.1161/01.ATV.17.4.741

Juhasz, S., Elbakry, A., Mathes, A., \& Lobrich, M. (2018). ATRX Promotes DNA Repair Synthesis and Sister Chromatid Exchange during Homologous Recombination. Molecular Cell , 71 (1), 11-24. e7.https://doi.org/10.1016/j.molcel.2018.05.014

Kashiwagi, K., Kondo, S., Yoshida, W., \& Yoshioka, M. (2000). Effects of temperature and salinity on hatching success of Japanese whitingSillago japonica eggs. Suisanzoshoku , 48 (4), 637642.https://doi.org/10.11233/aquaculturesci1953.48.637

Kawahata, H., \& Ohshima, H. (2004). Vegetation and environmental record in the northern 
East China Sea during the late Pleistocene. Global and Planetary Change , 41 (3/4), 251273.https://doi.org/10.1016/j.gloplacha.2004.01.011

Kawate, H., Itoh, R., Sakumi, K., Nakabeppu, Y., Tsuzuki, T., Ide, F., .. Sekiguchi, M. (2000). A defect in a single allele of the Mlh1 gene causes dissociation of the killing and tumorigenic actions of an alkylating carcinogen in methyltransferase-deficient mice.Carcinogenesis , 21 (2), 301305.https://doi.org/10.1093/carcin/21.2.301

Lamichhaney, S., Fuentes-Pardo, A. P., Rafati, N., Ryman, N., McCracken, G. R., \& Bourne, C. (2017). Parallel adaptive evolution of geographically distant herring populations on both sides of the North Atlantic Ocean. Proceedings of the National Academy of Sciences of The United States of America , 114 (17), E3452-E3461.https://doi.org/10.1073/pnas.1617728114

Leonard, W. R., Snodgrass, J. J., \& Sorensen, M. V. (2005). Metabolic adaptations in indigenous Siberian populations. Annual Review of Anthropology , 34 (1), 451471.https://doi.org/10.1146/annurev.anthro.34.081804.120558

Li, H., \& Durbin, R. (2009). Fast and accurate short read alignment with Burrows-Wheeler transform. Bioinformatics , 25 (14), 1754-1760.https://doi.org/10.1093/bioinformatics/btp324

Li, H., \& Durbin, R. (2011). Inference of human population history from individual whole-genome sequences. Nature , 475 (7357), 493-496.https://doi.org/10.1038/nature10231

Li, H., Handsaker, B., Wysoker, A., Fennell, T., \& Ruan, J. (2009). The sequence alignment/map format and SAMtools. Bioinformatics ,25 (16), 2078-2079.https://doi.org/10.1093/gbe/evz118

Li, Y. L., Xue, D. X., Zhang, B. D., \& Liu, J. X. (2018). Population genomic signatures of genetic structure and environmental selection in the catadromous roughskin sculpin Trachidermus fasciatus. Genome Biology and Evolution , 11 (7), 1751-1764.https://doi.org/10.1093/gbe/evz118

Li, Z., Yu, L., Zhang, Y., Gao, J., Zhang, P., Wan, B., .. Zhao, S. (2001). Identification of human, mouse and rat PPP1R14A, protein phosphatase-1 inhibitor subunit 14A, \& mapping human PPP1R14A to chromosome 19q13.13-q13.2. Molecular Biology Reports ,28 (2), 91-101.https://doi.org/10.1023/a:1017998029053

Lisby, M., Barlow, J. H., Burgess, R. C., \& Rothstein, R. (2004). Choreography of the DNA damage response: Spatiotemporal relationships among checkpoint and repair proteins. Cell , 118 (6), 699713.https://doi.org/10.1016/j.cell.2004.08.015

Liu, B. J., Zhang, B. D., Xue, D. X., Gao, T. X., \& Liu, J. X. (2016). Population structure and adaptive divergence in a high gene flow marine fish: The small yellow croaker (Larimichthys polyactis ).PLoS ONE , 11 (4), e0154020.https://doi.org/10.1371/journal.pone.0154020

Liu, L. L., Zhu, H., Yan, Y. C., Wang, X. W., Zhang, R., \& Zhu, J. Y. (2018). Research Progress of Cold Tolerance Mechanism and Functional Genes in Fish. Biotechnology Bulletin.

Masutani, C., Kusumoto, R., Yamada, A., Dohmae, N., Yokoi, M., Yuasa, M., ... Hanaoka, F. (1999). The XPV (xeroderma pigmentosum variant) gene encodes human DNA polymerase eta. Nature , 399 (6737), 700-704.https://doi.org/10.1038/21447

McKay, R. J. (1992). Sillaginid fishes of the world (Family Sillaginidae). Food and Agriculture Organization of the United Nations Press, Italy.

Meer, G. V., Voelker, D. R., \& Feigenson, G. W. (2008). Membrane lipids: Where they are and how they behave. Nature Reviews Molecular Cell Biology , 9 (2), 112-124.https://doi.org/10.1038/nrm2330

Oka, O. B. V., Pringle, M. A., Schopp, I. M., Braakman, I., \& Bulleid, N. J. (2013). ERdj5 Is the ER Reductase that Catalyzes the Removal of Non-Native Disulfides and Correct Folding of the LDL Receptor.Molecular Cell , 50 (6), 793-804.https://doi.org/10.1016/j.molcel.2013.05.014 
Oozeki, Y., Hwang, P. P., \& Hirano, R. (1992). Larval development of the Japanese whiting, Sillago japonica . Japan J. Ichthyol.39 (1), 59-66.

Oshima, S., Nakamura, T., Namiki, S., Okada, E., Tsuchiya, K., Okamoto, R., ... Watanabe, M. (2004). Interferon Regulatory Factor 1 (IRF-1) and IRF-2 Distinctively Up-Regulate Gene Expression and Production of Interleukin-7 in Human Intestinal Epithelial Cells. Molecular and Cellular Biology , 24 (14), 6298-6310.https://doi.org/10.1128/mcb.24.14.6298-6310.2004

Park, H. G., Han, S. I., Oh, S. Y., \& Kang, H. S. (2005). Cellular responses to mild heat stress. Cellular and Molecular Life Sciences, 62 (1), 10-23.https://doi.org/10.1007/s00018-004-4208-7

Phillips, S. J., Anderson, R. P., \& Schapire, R. E. (2006). Maximum entropy modeling of species geographic distributions. Ecological Modelling, 190 (2006), 231-259.https://doi.org/10.1016/j.ecolmodel.2005.03.026

Pickrell, J. K., \& Pritchard, J. K. (2012). Inference of population splits and mixtures from genome-wide allele frequency data. PLoS Genetics , 8 (11), e1002967.https://doi.org/10.1371/journal.pgen.1002967

Purcell, S., Neale, B., Todd-Brown, K., Thomas, L., Ferreira, M. A. R., Bender, D., ... Sham, P.C. (2007). PLINK: A Tool Set for Whole-Genome Association and Population-Based Linkage Analyses.American Journal of Human Genetics , 81 (3), 559-575.https://doi.org/10.1086/519795

Rouhiainen, A., Zhao, X., Vanttola, P., Qian, K., Kulesskiy, E., Kuja-Panula, J., ... Rauvala, H. (2016). HMGB4 is expressed by neuronal cells and affects the expression of genes involved in neural differentiation. Scientific Reports , 6 , 32960.http://doi.org/10.1038/srep32960

Russell, N. J., \& Nichols, D. S. (1999). Polyunsaturated fatty acids in marine bacteria-a dogma rewritten. Microbiology , 145 (4), 767-779.https://doi.org/10.1007/s00253-018-9063-9

Saadeldin, I. M., Swelum, A. A., Elsafadi, M., Mahmood, A., Osama, A., Shikshaky, H., ... Magdeldin, S. (2020). Thermotolerance and plasticity of camel somatic cells exposed to acute and chronic heat stress. Journal of Advanced Research, 22 , 105-118.https://dx.doi.org/10.1016\%2Fj.jare.2019.11.009

Schluter, D. (2000). Ecological character displacement in adaptive radiation. American Naturalist, 156 (4), S4-S16.https://doi.org/10.1086/303412

Skelly, D. K., Joseph, L. N., Possingham, H. P., Freidenburg, L. K., Farrugia, T. J., Kinnison, M. T., \& Hendry, A. P. (2007). Evolutionary responses to climate change. Conservation Biology , 21 (5), 13531355.https://doi.org/10.1111/j.1523-1739.2007.00764.x

Spivakov, M., Auer, T. O., Peravali, R., Dunham, I., Dolle, D., Fujiyama, A., ... Birney, E. (2014). Genomic and phenotypic characterization of a wild medaka population: towards the establishment of an isogenic population genetic resource in fish. G3-Genes Genomes Genetics , 4 (3), 433445.https://doi.org/10.1534/g3.113.008722

Wang, J., Xue, D. X., Zhang, B. D., Li, Y. L., Liu, B. J., \& Liu, J. X. (2016). Genome-wide SNP discovery, genotyping and their preliminary applications for population genetic inference in spotted sea bass (Lateolabrax maculatus ).PLoS ONE , 11 (6), e0157809.https://doi.org/10.1371/journal.pone.0157809

Wang, K., Li, M., \& Hakonarson, H. (2010). ANNOVAR: functional annotation of genetic variants from highthroughput sequencing data.Nucleic Acids Research , 38 (16), e164.https://doi.org/10.1093/nar/gkq603

White, C. R., Alton, L. A., \& Frappell, P. B. (2012). Metabolic cold adaptation in fishes occurs at the level of whole animal, mitochondria and enzyme. Proceedings of the Royal Society B-Biological Sciences , 279 (1734), 1740-1747.https://doi.org/10.1098/rspb.2011.2060

Xia, S., Xiao, L., Gannon, P., \& Li, X. (2010). RFC3 regulates cell proliferation and pathogen resistance in Arabidopsis. Plant Signaling \& Behavior , 5 (2), 168-170.https://doi.org/10.4161/psb.5.2.10526 
Xu, J., Li, J. T., Jiang, Y. L., Peng, W. Z., Yao, Z. L., Chen, B. H., .. Xu, P. (2017). Genomic basis of adaptive evolution: The survival of Amur Ide (Leuciscus waleckii ) in an Extremely Alkaline Environment. Molecular Biology and Evolution ,34 (1), 145-159.https://doi.org/10.1093/molbev/msw230

Xu, S. Y., Song, N., Zhao, L. L., Cai, S. S., Han, Z. Q., \& Gao, T. X. (2017). Genomic evidence for local adaptation in the ovoviviparous marine fish Sebasticcus marmoratus with a background of population homogeneity. Scientific Reports , 7 (1), 1562.https://doi.org/10.1038/s41598-017-01742-z

Xue, T. Q., Du, N., \& Gao, T. X. (2010). Phylogenetic relationships of 4 Sillaginidae species based on partial sequences of COI and cytochrome b gene. Journal of Ocean University China , 40 (S1), 91-98.

Yamada, K., Ono, M., Perkins, N. D., Rocha, S., \& Lamond, A. I. (2013). Identification and Functional Characterization of FMN2, a Regulator of the Cyclin-Dependent Kinase Inhibitor p21. Molecular Cell ,49 (5), 922-933.https://doi.org/10.1016/j.molcel.2012.12.023

Yang, H., Mergler, S., Sun, X. C., Wang, Z., Lu, L., Bonanno, J. A., ... Reinach, P. S. (2005). TRPC4 knockdown suppresses epidermal growth factor-induced store-operated channel activation and growth in human corneal epithelial cells. Journal of Biological Chemistry ,280 (37), 3223032237.https://doi.org/10.1074/jbc.m504553200

Yang, J., Lee, S. H., Goddard, M. E., \& Visscher, P. M. (2011). GCTA: a tool for Genome-wide Complex Trait Analysis. American Journal of Human Genetics , 88 (1), 76-82.https://doi.org/10.1016/j.ajhg.2010.11.011

Yang, T. Y., Gao, T. X., Meng, W., \& Jiang, Y. L. (2020). Genome-wide population structure and genetic diversity of Japanese whiting (Sillago japonica) inferred from genotyping-by-sequencing (GBS): Implications for fisheries management. Fisheries Research , 225, 105501.https://doi.org/10.1016/j.fishres.2020.105501

Zhang, Z., Xu, S., Capinha, C., Weterings, R., \& Gao, T. (2019). Using species distribution model to predict the impact of climate change on the potential distribution of Japanese whiting Sillago japonica .Ecological Indicators , 104 (SEP.), 333-340.https://doi.org/10.1016/j.ecolind.2019.05.023

\section{Figure legend}

Figure 1 (a) Map of sampling locations. Annual sea surface temperature is indicated. (b) Genome-wide distribution of nucleotide diversity in $40 \mathrm{~kb}$ non-overlapping windows.

(c) Admixture analysis of the five Sillago japonica populations. The length of each colored segment represents the proportion of the individual genome inferred from ancestral populations $(K=2-6)$. (d) Principal components 1 and 2 for the 49 Sillago japonicaindividuals. (e) Neighbor-Joining tree constructed using p-distances among the 49 Sillago japonica individuals.

Figure 2 Plot of pairwise estimates of $F \mathrm{ST} /(1-F \mathrm{ST})$ versus two types of geographic distances (coastal distance and oceanic distance) between the populations.

Figure 3 Demographic history for each population inferred from PSMC analysis.

Figure 4 Pattern of population splits and mixture between the fiveSillago japonica populations. The drift parameter is proportional to Ne generations, where Ne is the effective population size. Scale bar shows the average standard error of the estimated entries in the sample covariance matrix.

Figure 5 Genomic regions with strong selective signals in populations ofSillago japonica. (a) Distribution of $\log _{2}\left(\vartheta_{\pi}\right.$ ratios $)$ and $F$ ST values calculated in $40 \mathrm{~kb}$ sliding windows with $20 \mathrm{~kb}$ increments between the RS/ZS populations (ZS as control group). The data points in red (corresponding to the top 5\% of empirical $\log _{2}\left[\vartheta_{\pi}\right.$ ratios $]$ ratio distribution with values of $>0.1204$ and the top $5 \%$ of $F$ ST distribution with values of $>0.0904$ ) are genomic regions under selection in the RS population. (b) Overlap candidate genes of the RS/ZS and RS/ST pairs based on a Venn diagram. (c) Overlap candidate genes of the ST/ZS and ST/RS pairs based on a Venn diagram. (d) Overlap candidate genes of the ZS/RS and Japan/RS pairs based on a Venn diagram. (e) Allele frequency of one SNP within the cold-temperature adaptation genePicalm across 
the five $S$. japonica populations. (f) Allele frequency of one SNP within the high-temperature adaptation geneARHGAP42 across the five S. japonica populations. (g) Allele frequencies of one SNP within the warm-temperature adaptation gene SORCS3 across the five $S$. japonica populations.

Figure 6 PCA based on the SNPs located in the candidate genes (a, cold-temperature adaptation genes; b, high-temperature adaptation genes; c, warm-temperature adaptation genes).

Figure 7 Top 20 KEGG pathway enrichment statistics for the candidate genes.

Figure 8 The predicted potential distribution (a, b), changes in habitat suitability (c) of China group under RCP45 scenarios and (d) response curves of predicted occurrence probability of China group against temperature.

Figure 9 The predicted potential distribution (a, b), changes in habitat suitability (c) of Japan group under RCP45 scenarios and (d) response curves of predicted occurrence probability of Japan group against temperature. 
(a)

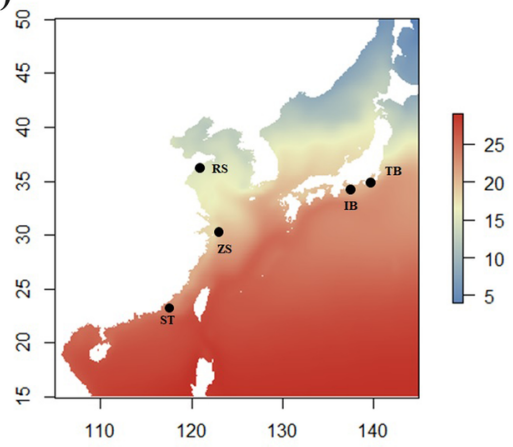

(c)

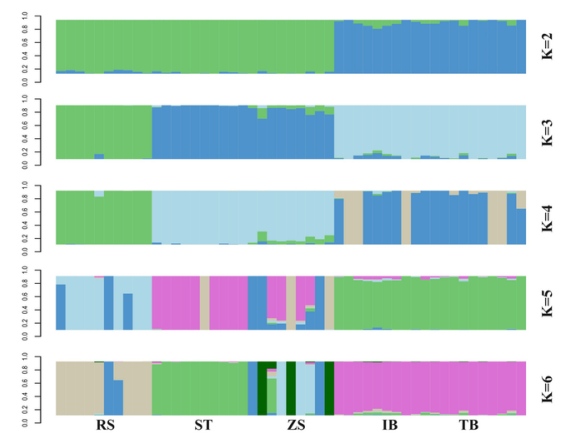

(e)

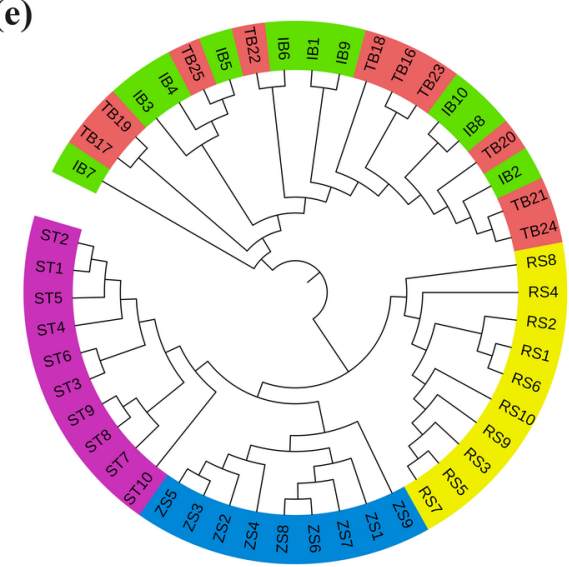

(b)

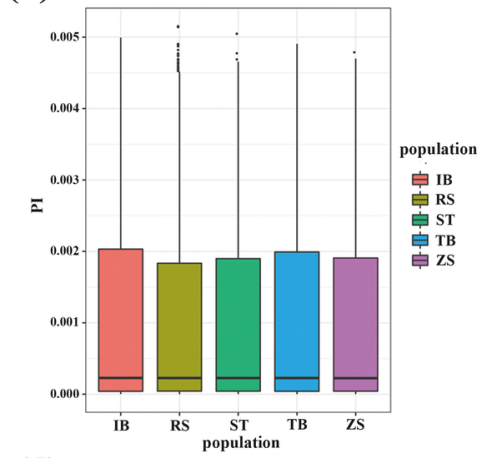

(d)

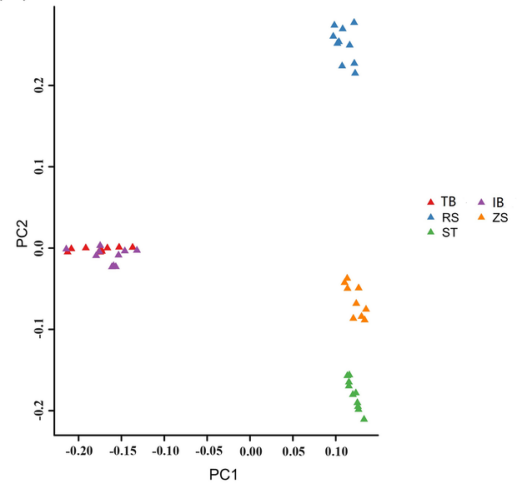



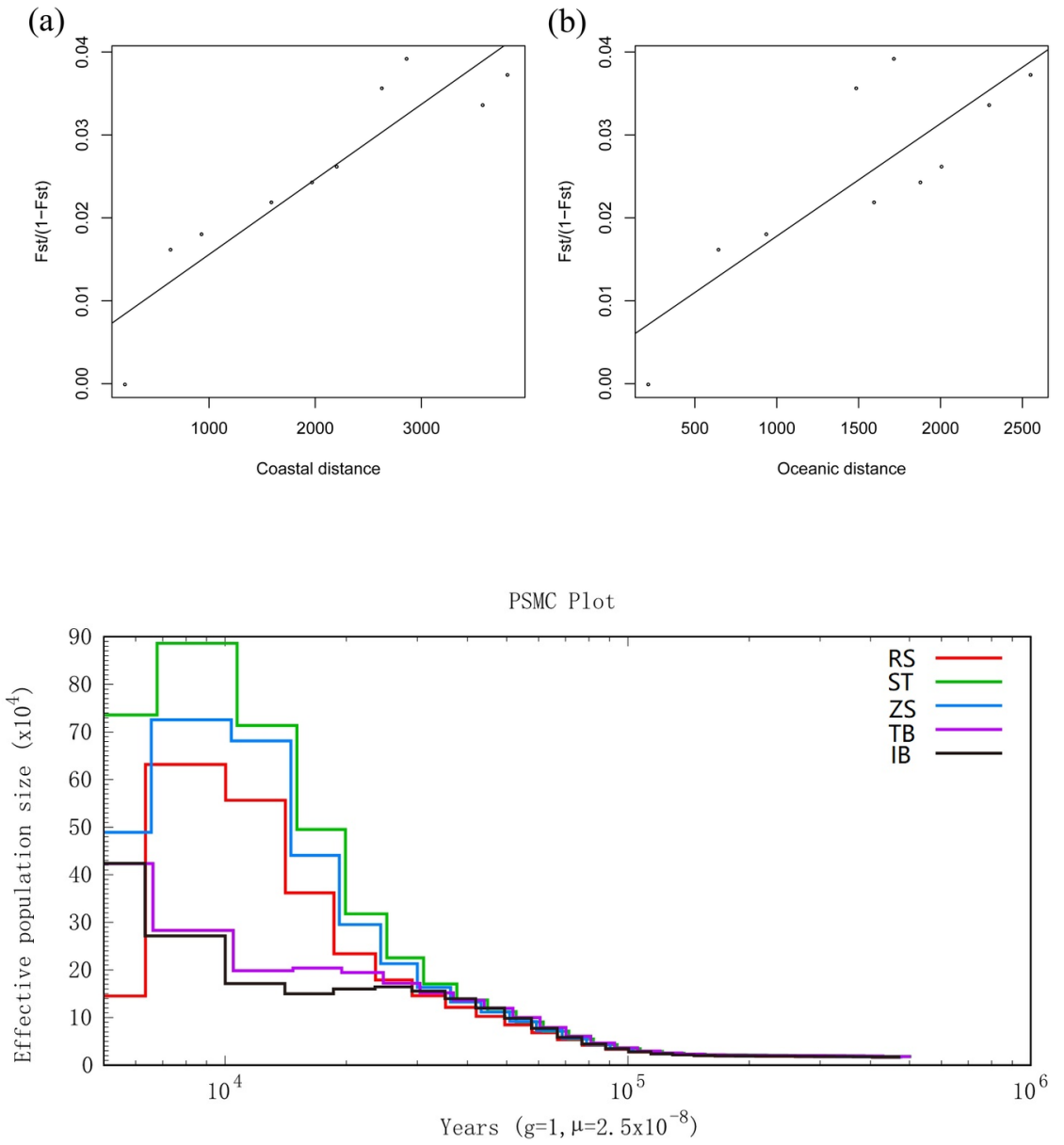

(a)

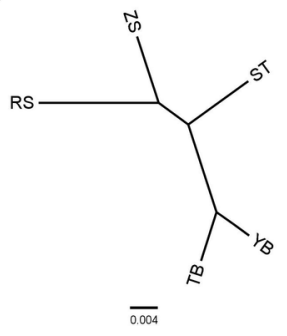

(b)

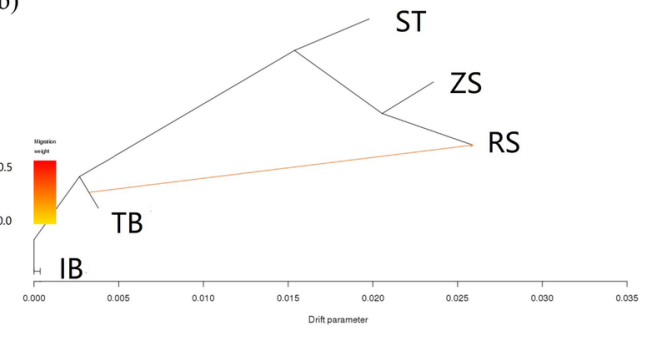



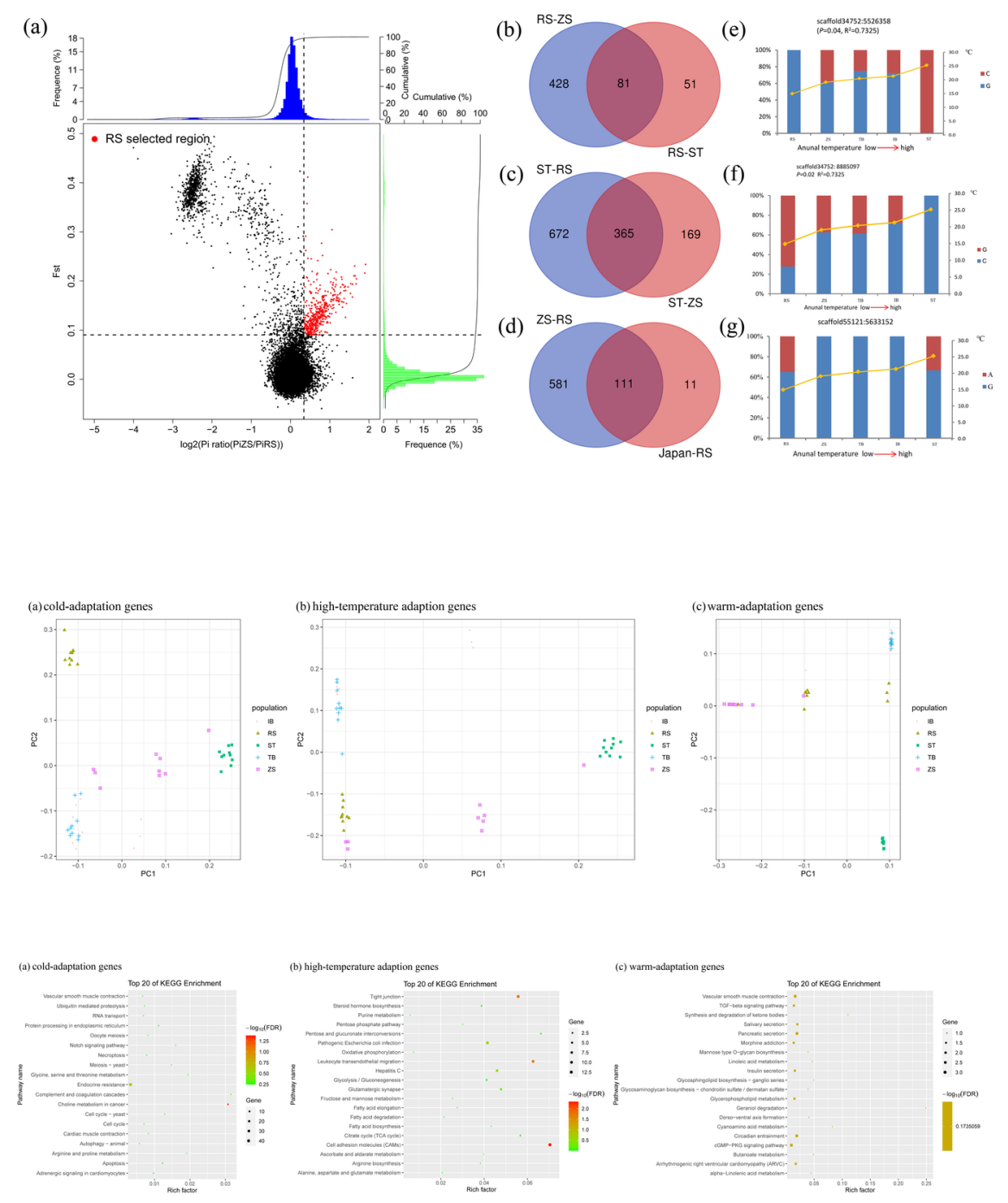
(a) Predicted current potential distribution

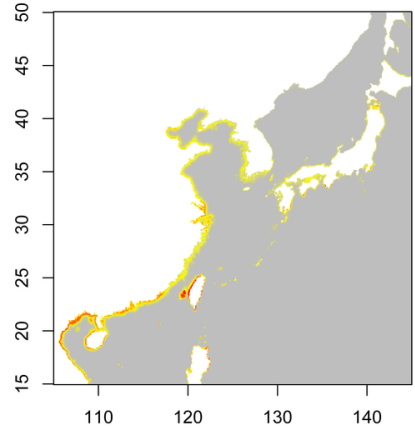

(c) Change under 2100RCP4.5

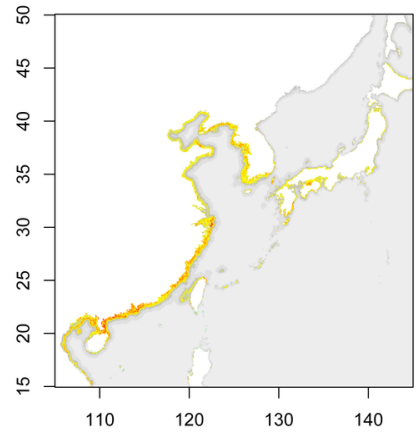

(a) Predicted current potential distribution

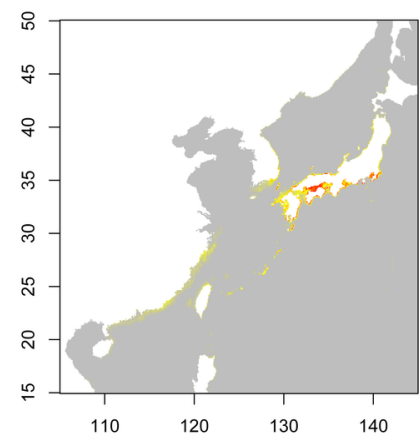

(c) Change under 2100RCP4.5

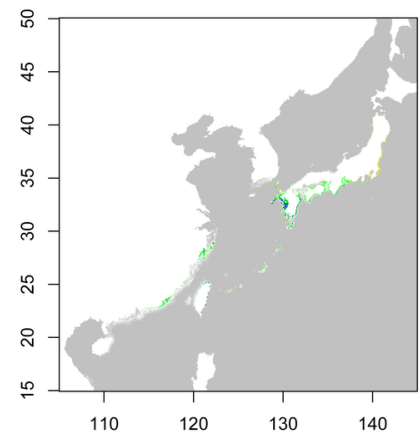

(b) Distribtuion under 2100RCP4.5

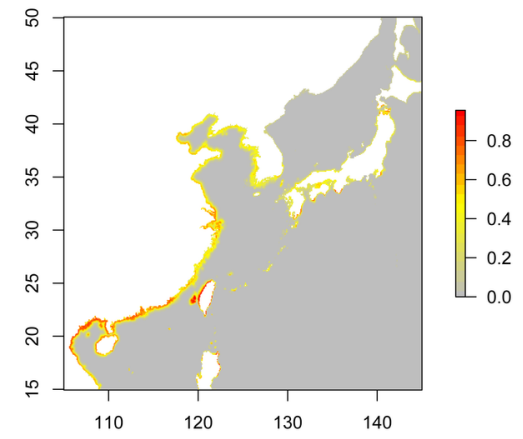

(d) Response curve against temperature

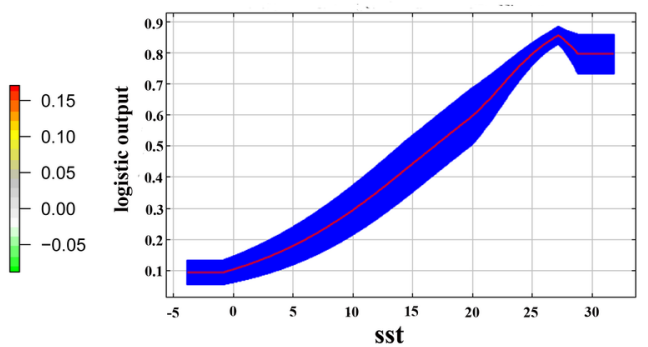

(b) Distribtuion under 2100RCP4.5

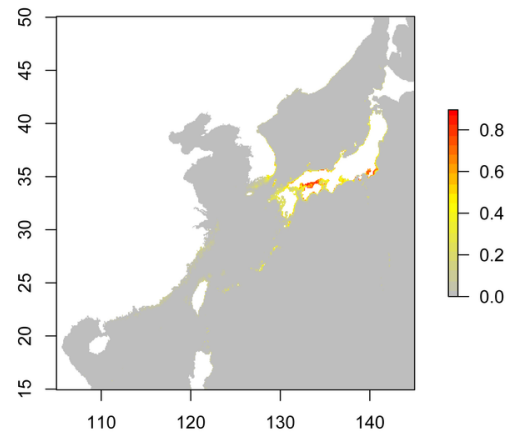

(d) Response curve against temperature

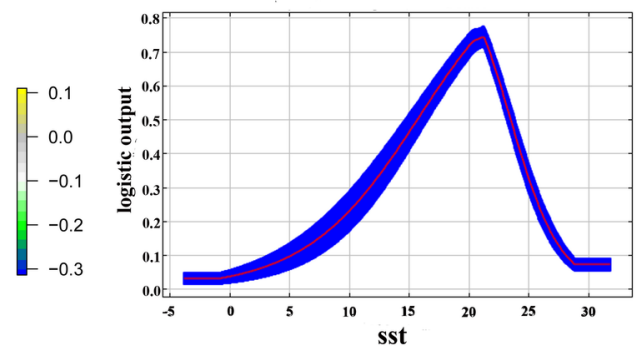

Hosted file 
Table1.pdf available at https://authorea.com/users/365476/articles/508129-paralleladaptations-of-japanese-whiting-sillago-japonica-under-temperature-stress

\section{Hosted file}

Table2.pdf available at https://authorea.com/users/365476/articles/508129-paralleladaptations-of-japanese-whiting-sillago-japonica-under-temperature-stress 